\title{
Plasma Glial Fibrillary Acidic Protein Levels Differ Along the Spectra of Amyloid Burden and Clinical Disease Stage
}

\author{
Breton M. Asken, Fanny M. Elahi, Renaud La Joie, Amelia Strom, Adam M. Staffaroni, \\ Cutter A. Lindbergh, Alexandra C. Apple, Michelle You, Sophia Weiner-Light, Nivetha Brathaban, \\ Nicole Fernandes, Anna Karydas, Paul Wang, Julio C. Rojas, Adam L. Boxer, Bruce L. Miller, \\ Gil D. Rabinovici, Joel H. Kramer and Kaitlin B. Casaletto
}

[Journal of Alzheimer's Disease 78(1), 2020, 265-276, DOI 10.3233/JAD200755]

https://content.iospress.com/articles/journal-of-alzheimers-disease/jad200755

After publication of the article, the authors were alerted to a minor error with the amyloid PET SUVR-toCentiloids conversion equation for the subset of participants scanned with the Pittsburgh Compound B tracer. The error involved the constant term in the conversion equation (not the multiplier). The authors quickly reanalyzed the relevant data and here below note the changes to some of the statistical outcomes in the study. There were no changes warranting updated interpretations/discussion points.

In the Abstract: Results section on p. 265, the bold text has changed from the original publication:

Results: In both cohorts, plasma GFAP increased linearly with A $\beta$-PET CLs in clinically normal older adults. In Cohort 2, which included participants with more severe clinical dysfunction and A $\beta$-PET burden, the association between $A \beta$ and GFAP became curvilinear (inverted U-shape; quadratic model $\mathbf{R}^{\mathbf{2}}$ change $=. \mathbf{1 5 8}, \mathbf{p}=\mathbf{. 0 1}$ ), and $A \beta-P E T$ interacted with CDR-SB $\left(\mathbf{R}^{2}\right.$ change $\left.=\mathbf{. 1 6 9}, \mathbf{p}=\mathbf{. 0 0 6}\right)$ : older adults with intermediate functional impairment (CDR-SB=0.5-4.0) showed a weak (negative) association between A $\beta$-PET CLs and plasma GFAP, while older adults with dementia (CDR-SB > 4.0) showed a strong, negative association of higher A $\beta$-PET CLs with lower plasma GFAP.

In the METHODS: Amyloid PET Imaging section on p. 267-268, the bold text has changed from the original publication:

Amyloid PET Imaging

Cortical A $\beta$ deposition was defined by PET completed with either ${ }^{11} \mathrm{C}$-Pittsburgh compound $\mathrm{B}(\mathrm{PiB} ; \mathrm{N}=22$ [60\%] of Cohort 2 participants) or ${ }^{18} \mathrm{~F}$-florbetapir $(\mathrm{N}=50$ [100\%] of Cohort $1, \mathrm{~N}=15$ [40\%] of Cohort 2). Standardized uptake value ratios (SUVR) were calculated and then converted to the Centiloids (CLs) scale to harmonize data across the two tracers [22]. The Centiloids conversion is used to calibrate A $\beta$-PET measurements acquired with different tracers and analytic pipelines to a common scale. A value of $100 \mathrm{CLs}$ corresponds with the average degree of $A \beta$ deposition observed on PET imaging in patients diagnosed with mild-moderate Alzheimer's dementia, while a value of 0 CLs corresponds to mean uptake in healthy young controls devoid of A $\beta$ pathology [22]. We used continuous CL values for regression analyses, but report A $\beta$-PET positivity frequencies based on processing pipeline- and tracer-specific thresholds: PiB SUVR > 1.21 (8.6 CLs); florbetapir SUVR > 1.11 (22.5 CLs). See Supplemental Methods for more details.

In the RESULTS: Cohort Demographics section on p. 268-269, the bold text has changed from the original publication: 


\section{Cohort Demographics}

Participant characteristics stratified by study cohort are summarized in Table 1 . There were no statistically significant differences between cohorts in age, sex, education, race, or frequency of APOE e4 carriers. As expected based on inclusion of more MCI and dementia participants, Cohort 2 had higher average A $\beta$-PET CLs (64.9 vs. $36.6 \mathrm{CLs}, \mathbf{p}=\mathbf{. 0 0 4}$ ), was more likely to be A $\beta$-PET positive (73\% vs. 50\%, $p=.03$ ), had worse global cognition (Mini Mental State Examination; median score 27 vs. 29, $p<.001$ ), and had higher CDR-SB scores (Figure $1 ; p<.001$ ). Plasma GFAP was not significantly associated with plasma $\mathrm{NfL}$ after controlling for age and sex in Cohort $1(\beta=0.175, p=.19)$ but showed a significant positive association in Cohort $2(\beta=0.584, p<.001)$. There was no significant difference in plasma GFAP concentrations between APOE e4 carriers and non-carriers in either Cohort $1(\mathrm{t}=0.978, p=.33)$ or Cohort $2(\mathrm{t}=-1.078, p=.29)$.

In the RESULTS: A $\beta$-PET Association with Plasma GFAP section on p.269, the bold text has changed from the original publication:

Aß-PET Association with Plasma GFAP

In Cohort 1 , higher A $\beta$-PET CLs was associated with higher plasma GFAP $(\beta=0.324, p=.01)$, with a slightly stronger effect seen when controlling for APOE e4 carrier status $(\beta=0.417, p=.005)$. Including the quadratic $\mathrm{A} \beta$-PET CLs term did not improve model fit (quadratic model $\mathrm{R}^{2}$ change $=.002, p=.75$ Figure $2 \mathrm{~A}$ ). In Cohort 2 , there was a statistically significant, negative curvilinear association between A $\beta$-PET CLs and plasma GFAP (Figure 2B). Including the quadratic A $\beta$-PET CLs term improved model fit (quadratic model $\mathbf{R}^{\mathbf{2}}$ change $=\mathbf{. 1 5 8}$, $\mathbf{p}=\mathbf{. 0 1})$ with both the linear $(\mathbf{p}=\mathbf{. 0 0 4})$ and quadratic $(\mathbf{p}=\mathbf{. 0 1})$ A $\beta$-PET CLs effects remaining statistically significant. The Cohort 2 scatterplot showed that plasma GFAP increased until around 75 CLs before plateauing and then decreasing beyond $100 \mathrm{CLs}$. Associations remained statistically significant when additionally controlling for APOE e 4 carrier status and plasma NfL concentration. For completeness, we also evaluated the A $\beta$-PET CLs $x$ plasma NfL and A $\beta$-PET CLs x APOE status interactions on plasma GFAP. There was no significant A $\beta$-PET CLs x plasma NfL (Cohort 1: $\mathrm{R}^{2}$ change $=.042, p=.10$; Cohort 2: $\mathbf{R}^{\mathbf{2}}$ change $=. \mathbf{0 0 2}, \mathbf{p}=. \mathbf{7 3}$ ) or A $\beta$-PET CLs x APOE (Cohort $1: \mathrm{R}^{2}$ change $=.006, p=.54$; Cohort $2: \mathbf{R}^{2}$ change $=. \mathbf{0 0 2}, \mathbf{p}=. \mathbf{8 1}$ ) association with plasma GFAP.

In the RESULTS: A $\beta$-PET Association with Plasma NfL section on p. 269-270, the bold text has changed from the original publication:

Aß-PET Association with Plasma NfL

In contrast, we did not observe a significant linear or nonlinear association between A $\beta$-PET CLs and plasma NfL in either Cohort 1 (linear $\beta=0.035, p=.78$, quadratic $\mathrm{R}^{2}$ change $=.008, p=.48$; Figure $2 \mathrm{C}$ ) or Cohort 2 (linear $\beta=0.215, p=.25$, quadratic $R^{2}$ change $=.055, p=.16$; Figure $2 D$ ).

In the RESULTS: Interactions of A $\beta-P E T$ and $C D R-S B$ section on p. 270-271, the bold text has changed from the original publication:

Interactions of $A \beta-P E T$ and $C D R-S B$

Given the observed nonlinear association between A $\beta$-PET CLs and plasma GFAP in Cohort 2, we investigated whether this was related to clinical disease stage by testing the A $\beta$-PET CLs x CDR-SB interaction effect on plasma GFAP. In Cohort 2 there was a statistically significant A $\beta$-PET CLs x CDR-SB interaction on plasma GFAP $\left(\mathbf{R}^{\mathbf{2}}\right.$ change $=\mathbf{. 1 6 9}, \mathbf{p}=\mathbf{. 0 0 6}$; Figure $\left.3 \mathrm{~B}\right)$, which remained after controlling for APOE e4 carrier status $\left(\mathbf{R}^{2}\right.$ change $=. \mathbf{2 0 4}, \mathbf{p}=\mathbf{. 0 0 3})$. Cohort 2 participants with $C D R-S B=0$ showed a strong, positive association of higher $\mathrm{A} \beta$-PET CLs with higher plasma GFAP (linear model $\mathbf{R}^{\mathbf{2}}=\mathbf{. 4 7 2}$ ), while Cohort 2 participants with CDR-SB $>4.0$ showed a strong, negative association of higher A $\beta$-PET CL with lower plasma GFAP (linear model $\mathbf{R}^{\mathbf{2}}=\mathbf{. 3 9 5}$ ). Cohort 2 participants with intermediate $\mathrm{CDR}-\mathrm{SB}=0.5-4.0$ showed a weak (negative) association between $\mathrm{A} \beta$-PET $\mathrm{CL}$ and plasma GFAP (linear model $\mathrm{R}^{2}=.090$ ). The interaction did not reach statistical significance in Cohort $1\left(\mathrm{R}^{2}\right.$ change $=.023, p=.23$ ) but showed similar directionality (Figure 3A). Cohort 1 participants with CDR$\mathrm{SB}=0$ showed a positive association of higher $\mathrm{A} \beta$-PET CLs with higher plasma GFAP (linear model $\mathrm{R}^{2}=.090$ ) while participants with $\mathrm{CDR}-\mathrm{SB}=0.5-4.0$ showed little-to-no meaningful association (linear model $\mathrm{R}^{2}<.000$ ). Controlling for plasma NfL somewhat attenuated the A $\beta$-PET CL x CDR-SB interaction on plasma GFAP in Cohort $2\left(\mathrm{R}^{2}\right.$ change $\left.=.061, p=.05\right)$.

Again, in contrast, there was no significant A $\beta$-PET CLs x CDR-SB interaction on plasma NfL in either Cohort $1\left(\mathrm{R}^{2}\right.$ change $=.005, p=.59$; Figure $\left.3 \mathrm{C}\right)$ or Cohort $2\left(\mathbf{R}^{\mathbf{2}}\right.$ change $=\mathbf{. 0 6 9}, \mathbf{p}=\mathbf{. 1 2}$; Figure 3D; see also Supplementary Figure 1). Exploratory decomposition of the interaction terms suggested A $\beta$-PET CLs may be positively 
associated with plasma NfL in Cohort 2 participants with CDR-SB=0 (Pearson's $\mathbf{r}=. \mathbf{3 5}$ ). We plotted A $\beta$-PET CLs and plasma GFAP as a function of CDR-SB score in both Cohort 1 and Cohort 2 to help visualize potential divergence of these biomarkers across the functional spectrum. Regression analyses controlling for age and sex supported a positive, linear association between A $\beta$-PET CLs and CDR-SB (Cohort 1 linear model $\mathrm{R}^{2}=.367$, $p<.001$; Cohort 2 linear model $\mathbf{R}^{2}=.557, \mathbf{p}<. \mathbf{0 0 1}$ ) and curvilinear association between plasma GFAP and CDR-SB across a wider clinical disease spectrum (Cohort 2, linear model $\mathrm{R}^{2}=.112$, quadratic model $\mathrm{R}^{2}=.246$; $\mathrm{R}^{2}$ change $p=.001$; Figure 4 ).

In the RESULTS: Sex as a Biologic Variable section on p. 271, the bold text has changed from the original publication:

\section{Sex as a Biologic Variable}

There was no significant main effect of sex on plasma GFAP, controlling for age, in either Cohort 1 ( $\beta=0.123$, $p=.32)$ or Cohort $2(\beta=0.121, p=.49)$. There also was no significant $A \beta$-PET CLs x Sex interaction on plasma GFAP in either Cohort $1\left(\mathrm{R}^{2}\right.$ change $\left.=.001, p=.77\right)$ or Cohort $2\left(\mathbf{R}^{\mathbf{2}}\right.$ change $=. \mathbf{0 5 9}, \mathbf{p}=. \mathbf{2 1}$; Supplemental

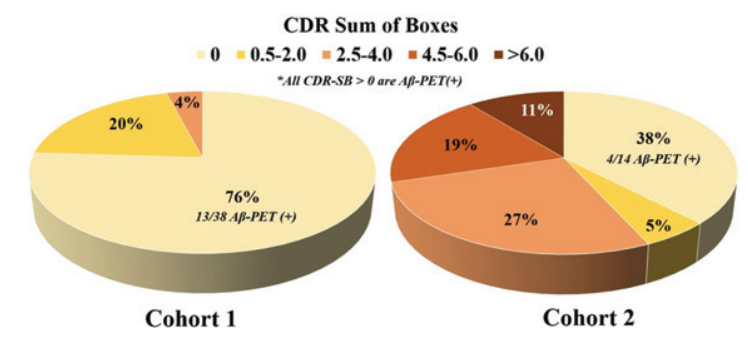

Figure 1. Clinical disease stage breakdown of Cohort 1 and Cohort 2.

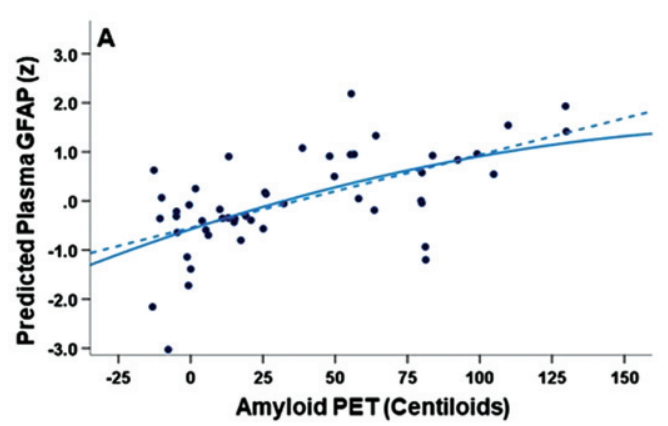

Cohort 1
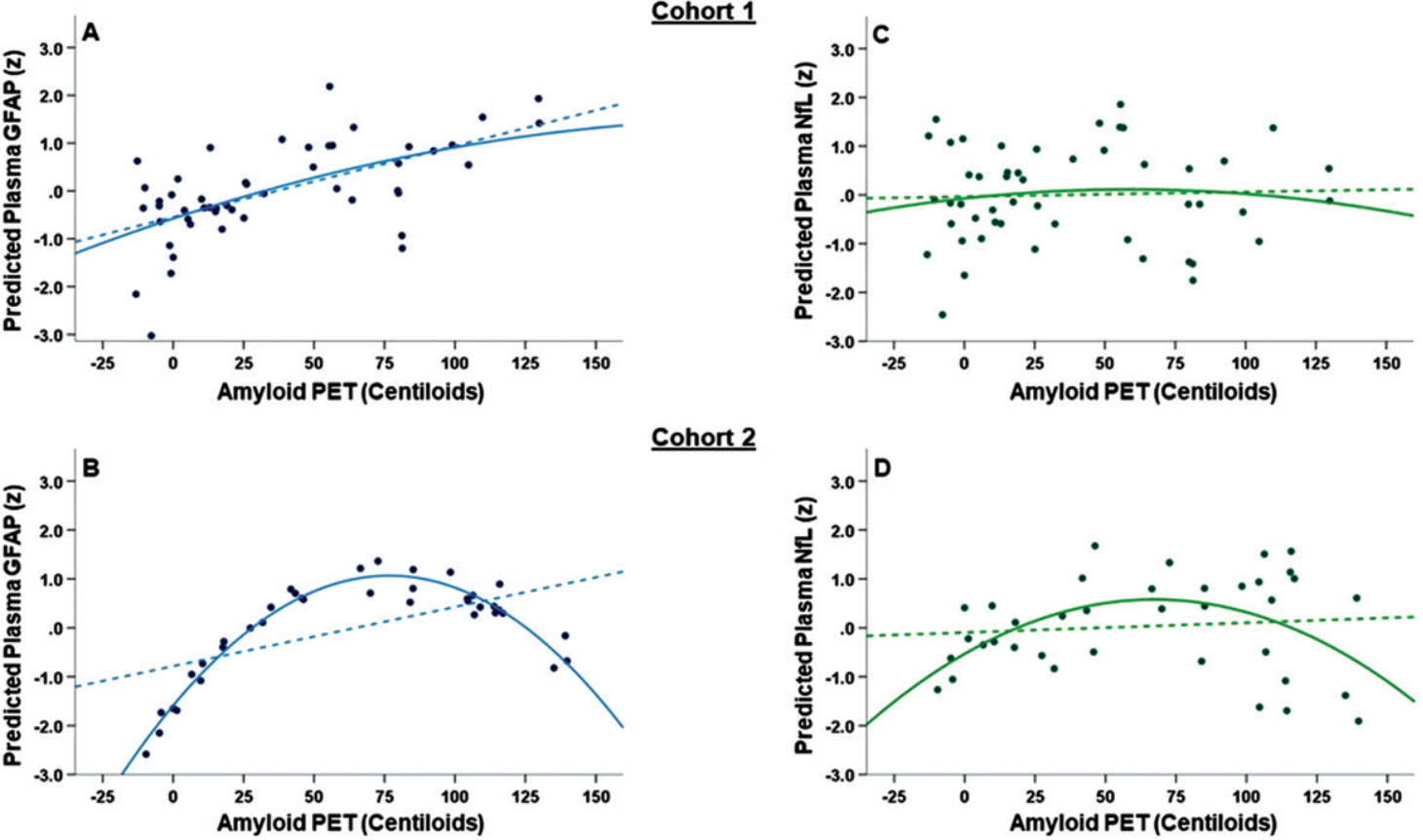

Cohort 2

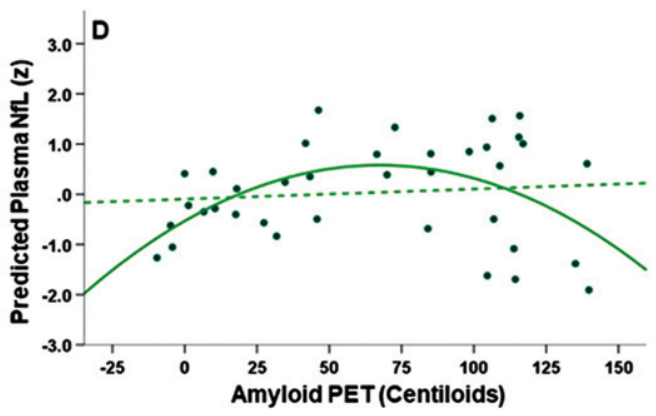

Figure 2. (A-D): Main effect of A $\beta$-PET CLs on plasma GFAP (A-B) and plasma NfL (C-D) concentrations. All figures show standardized model-predicted plasma biomarker concentrations on the Y-axis and both linear (dashed lines) and quadratic (solid lines) associations with amyloid PET (measured in Centiloids; CLs). For plasma GFAP, Cohort 1 (A) showed a linear association between higher A $\beta$-PET CLs and higher plasma GFAP while Cohort 2 (B) showed a curvilinear association. Cohort 2 notably differed from Cohort 1 in the range of clinical disease stage (see Figure 1). There were no statistically significant associations between Aß-PET CLs and plasma NfL in either Cohort 1 (C) or Cohort 2 (D). 
Figure 2). We found a trend towards statistical significance for a nonlinear A $\beta$-GFAP association in Cohort 2 females $\left(\mathbf{R}^{2}\right.$ change $\left.=.147, p=.11\right)$.

The four figures are reprinted below with minor alterations to the accurate amyloid-PET Centiloid values, along with updated figure captions where needed.
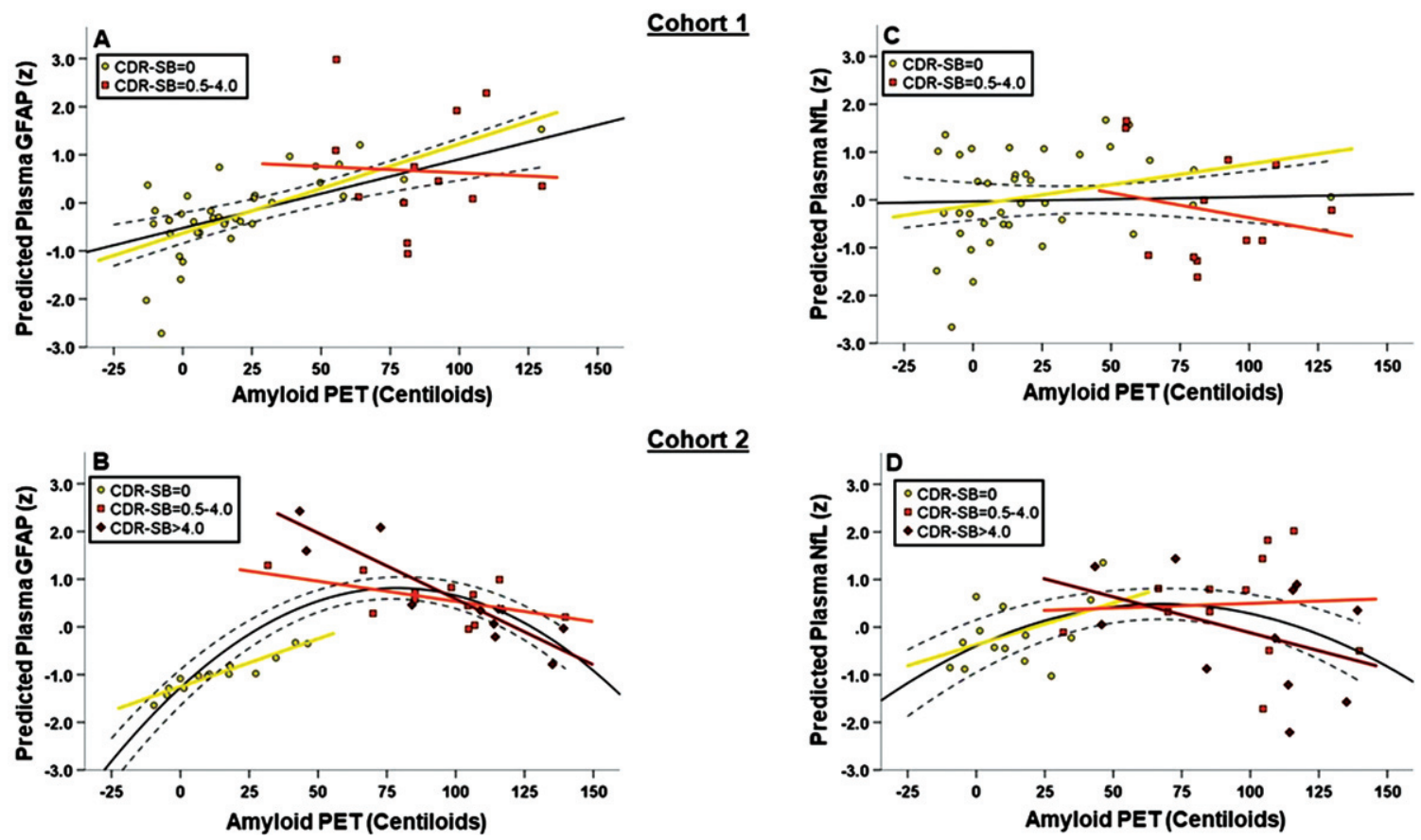

\section{Cohort 2}

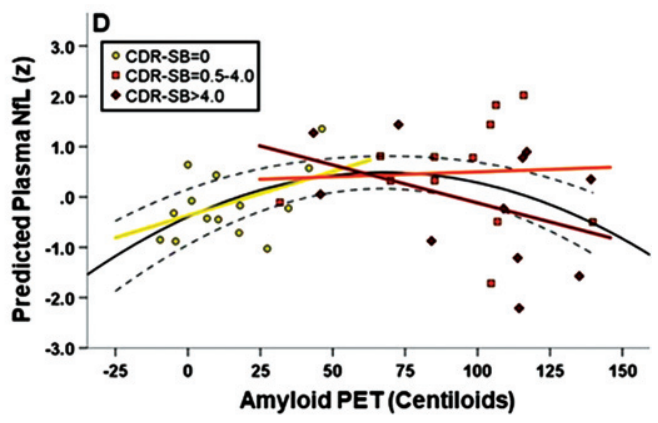

Figure 3. (A-D): Interaction between A $\beta$-PET and CDR-SB on plasma GFAP (A-B) and NfL (C-D) concentrations. All figures show standardized model-predicted plasma biomarker concentrations on the Y-axis. In both Cohort 1 (A) and Cohort 2 (B), higher A $\beta$-PET signal (measured in Centiloids; CLs) was associated with higher plasma GFAP in cases with CDR-SB=0 (yellow line) but not $\mathrm{CDR}-\mathrm{SB}=0.5-4.0$ (orange line). For cases in the dementia range (CDR-SB $>4.0$, red line, Cohort 2 only), higher A $\beta$-PET CLs was associated with lower plasma GFAP. The A $\beta$-PET CLs x CDR-SB interaction was statistically significant only in Cohort 2 for plasma GFAP. These associations largely were not observed for plasma NfL (C and D) except for an apparent association of higher A $\beta$-PET CLs with higher plasma NfL in Cohort 2 cases with $\mathrm{CDR}-\mathrm{SB}=0$ (Pearson's $\mathrm{r}=.40$ ).
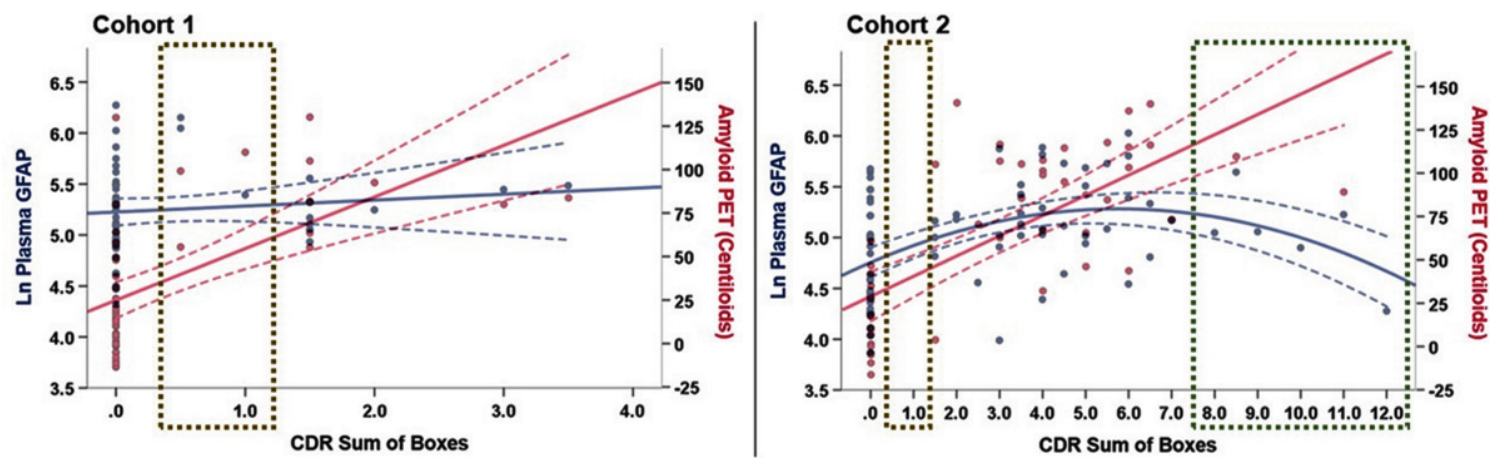

Figure 4. Plots depicting plasma GFAP (blue) and A $\beta$-PET (red) as a function of CDR-SB score. In Cohort 1 , plasma GFAP was not significantly associated with CDR-SB after controlling for age and sex $(\beta=.189, p=.16)$. In Cohort 2 , we observed potential divergence of plasma GFAP and $\mathrm{A} \beta$-PET in the in older adults with $\mathrm{CDR}-\mathrm{SB}>8.0$, though the $\mathrm{A} \beta$-PET data in this $\mathrm{CDR}$-SB range largely was extrapolated (green box). Cohorts notably differed in representation of older adults with CDR-SB>4.0 $(\mathrm{N}=0$ in Cohort 1) and in the mildest clinical disease stage (CDR-SB=0.5-1.0; gold boxes). NOTE: An additional 38 older adults from Cohort 2 with available plasma GFAP and CDR-SB (without A $\beta$-PET scan) were included in this plot to better represent the spectrum of clinical disease stage, particularly in the dementia range (total $\mathrm{N}=75$, age $=71.9 \pm 9.1$ years old, $52 \%$ female, $17.2 \pm 3.0$ years of education, $91.5 \%$ white). 